\title{
The ER - Glycogen Particle - Phagophore Triangle: A Hub Connecting Glycogenolysis and Glycophagy?
}

\section{József Mandl \& Gábor Bánhegyi}

Pathology \& Oncology Research Official Journal of the Arányi Lajos Foundation

ISSN 1219-4956

Volume 24

Number 4

Pathol. Oncol. Res. (2018) 24:821-826 DOI 10.1007/s12253-018-0446-0

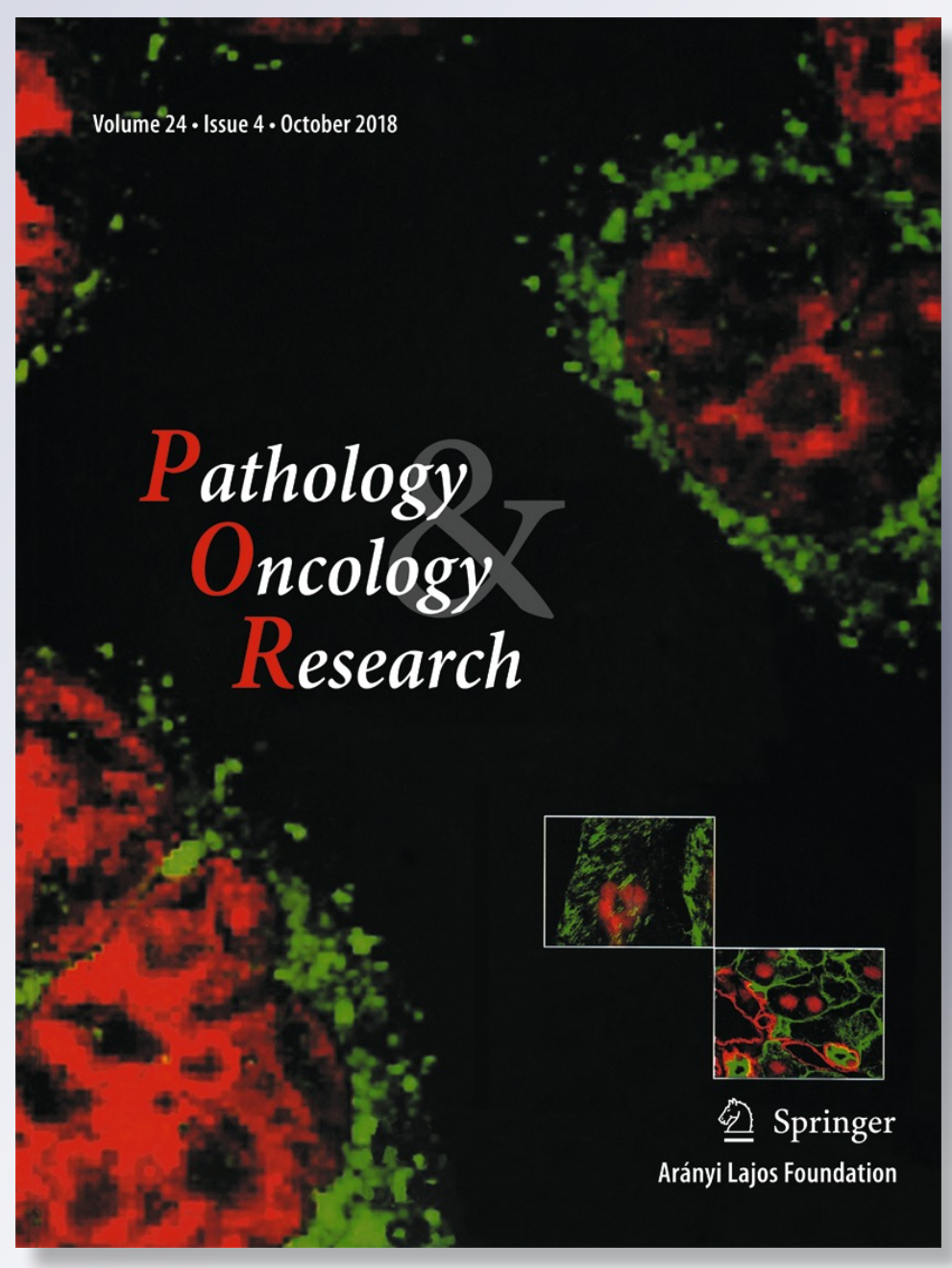

望 Springer 
Your article is protected by copyright and all rights are held exclusively by Arányi Lajos Foundation. This e-offprint is for personal use only and shall not be self-archived in electronic repositories. If you wish to self-archive your article, please use the accepted manuscript version for posting on your own website. You may further deposit the accepted manuscript version in any repository, provided it is only made publicly available 12 months after official publication or later and provided acknowledgement is given to the original source of publication and a link is inserted to the published article on Springer's website. The link must be accompanied by the following text: "The final publication is available at link.springer.com". 


\title{
The ER - Glycogen Particle - Phagophore Triangle: A Hub Connecting Glycogenolysis and Glycophagy?
}

\author{
József Mandl ${ }^{1} \cdot$ Gábor Bánhegyi $^{1}$ \\ Received: 4 April 2018 / Accepted: 28 June 2018 / Published online: 7 July 2018 \\ (C) Arányi Lajos Foundation 2018
}

\begin{abstract}
Glycogen particle is an intracellular organelle, which serves as a carbohydrate reserve in various cells. The function of glycogen is not entirely known in several cell types. Glycogen can be mobilized for different purposes, which can be related to cellular metabolic needs, intracellular redox state, metabolic state of the whole organism depending on regulatory aspects and also on cell functions. Essentially there are two different ways of glycogen degradation localized in different cellular organelles: glycogenolysis or lysosomal breakdown by acid alpha-glucosidase. While glycogenolysis occurs in glycogen particles connected to endoplasmic reticulum membrane, glycogen particles can be also combined with phagophores forming autophagosomes. A subdomain of the endoplasmic reticulum membrane - omegasomes - are the sites for phagophore formation. Thus, three organelles, the endoplasmic reticulum, the phagophore and the glycogen particle forms a triangle in which glycogen degradation occurs. The physiological significance, molecular logic and regulation of the two different catabolic paths are summarized and discussed with special aspect on the role of glycogen particles in intracellular organelle homeostasis and on molecular pathology of the cell. Pathological aspects and some diseases connected to the two different degradation pathways of glycogen particles are also detailed.
\end{abstract}

Keywords Glycogen particle · Glycogenolysis · Endoplasmic reticulum · Glycophagy · Lysosome · Phagophore

\section{Introduction}

Mobilization of energy reserves or storage of energy intake are fundamentally different states in metabolic regulation at cellular level. They can be connected either to actual state of the whole organism, but rather to actual metabolic conditions of the cell [1]. However, these metabolic controls are also dependent on cell types. Glycogen, as a branched polimer of glucose is an important energy depot, therefore a possible source of the immediate energy mobilization. Starvation is the most frequent indication for mobilization of energy reserves. The glycogen/tissue weight ratio is the highest in the liver. It is well known that liver glycogen is essentially for glucose supply in starvation to maintain blood glucose level. The glycogen/tissue weight ratio is smaller in skeletal muscle compared to liver. Muscle glycogen is mobilized mainly to cover

József Mandl

mandl.jozsef@med.semmelweis-univ.hu

1 Department of Medical Chemistry, Molecular Biology and Pathobiochemistry, Semmelweis University, Budapest, Hungary energy needs of cells for muscle contraction. However, glycogen can be found also in other tissues e.g. brain, erythrocytes, but its quantity is less compared to liver and muscle and little is known about its functions [1]. Nevertheless even neuronal glycogen breakdown is connected to energy mobilization for various cell specific purposes as learning, stress etc. [2].

Glycogen is not only a huge glucose store, but glycogen particle is also a cellular organelle. In spite of the fact that several moieties of glycogen molecule have been recognized long time ago, it is noteworthy that some important facts concerning on its functions, regulatory aspects and even certain constituents of the glycogen particle (e.g. glycogenin) have been explored relatively lately [2] .

For the integration of metabolic homeostasis at cellular level direct organelle-organelle connections are required. Organelles, as separate metabolic compartments are connected to each other in different ways, among others by transporters associated with integral membrane protein enzyme complexes. Organelle interplay among the endoplasmic reticulum (ER) and mitochondria is probably the most intensively studied connection, which underlies cellular metabolic homeostasis. Direct morphological and functional interactions 
for example between ER and mitochondria at membrane level are well documented $[3,4]$. The cross talk between ER and mitochondria is mainly mediated by mitochondria-associated membrane (MAM). Glycogen particles also participate in organelle-organelle interactions. Previously we have already published a hypothesis paper on physical and metabolic connections among glycogen particles and ER in hepatocytes [5]. We suggested the term ,glycogenoreticular system" for the description of this functional coupling between the two organelles. While recent proteomic data [6] confirm our hypothesis on the importance of ER-glycogen particle connections, new observations indicate a role of glycogen particles in autophagy, which is also an important possibility for intracellular nutrient and energy mobilization. These recent findings have focused attention to connection between glycogen particle and lysosome besides its previously known connections with ER (Fig. 1). Furthermore it turned out, that the two metabolic ways of glycogen degradation - glycogenolysis and acidic hydrolysis - and their different morphological localisations may be also important aspects of intracellular metabolic homeostasis. Recent advances in proteome analyses further contribute to the current view on the role of glycogen particles in intracellular metabolic integration. Our aim was to summarize the morphological and functional interplay between glycogen particles and cellular endomembrane system.

\section{Glycogen Particles for Glucose Supply to Intraluminal Formation of Various Metabolites in the ER}

ER is a metabolic compartment involved in a series of cellular functions require direct connections with other organelles.
Mitochondria are often referred as „energy powerhouse” of the cell, while ER has rather biosynthetic functions [7]. Molecules formed and matured in ER luminal compartment are usually secreted to the extracellular space [8]. Several precursors for various ER products are originated from glycogenolysis. Demand for mobilization of certain metabolites secreted from the ER are initiated by extracellular stimuli (e.g. decrease in blood glucose level in hepatocytes). But intracellular metabolic conditions (e.g. altered redox homeostasis due to enhanced mitochondrial metabolism in cardiomyocytes) may also stimulate mobilization of cellular energy reserves. Metabolism of liver glycogen is mainly controlled by extracellular stimuli depending on the actual state of metabolism in the organism, while mobilization of muscle glycogen stores is controlled rather ,inside" depending on the actual energetic state of the muscle cell.

Less attention has been focused on connection among ER and glycogen particles from the point of the maintenance of various ER functions, while the connection is morphologically proven $[9,10]$. Glucose obviously is in the center of metabolism both at the level of the organism and also at cellular level. The basic role of blood glucose level in the maintenance of metabolic homeostasis in mammals is related to its essential function in intracellular metabolism. Glycogen particles are participating in supply of several glucose derived metabolites to maintain intracellular metabolic homeostasis, and the bulk of these processes is localized in the ER.

Both glucose secretion and glucuronidation of endo- and xenobiotics in second phase of biotransformation in the liver are dependent on hepatic glycogen reserves [11, 12]. Thus, physical proximity results in direct metabolic interactions among glycogen particle and ER. A common character of these ER membrane embedded metabolic pathways is that
Fig. 1 Degradation of glycogen depends on organelle glycogen particle interactions. Glycogenolysis results in glucose formation, which is further metabolized or secreted from the cells depending also on the localization of glycogen berakdown. Glycogen particle can be connected to ER, or to lysosomes. ER:endoplasmic reticulum, G-6-P-ase:glucose-6phosphatase, UGT:UDPglucuronosyl transferase, GLO:gulonolactone oxidase, STBD1: starch binding domain containing protein 1

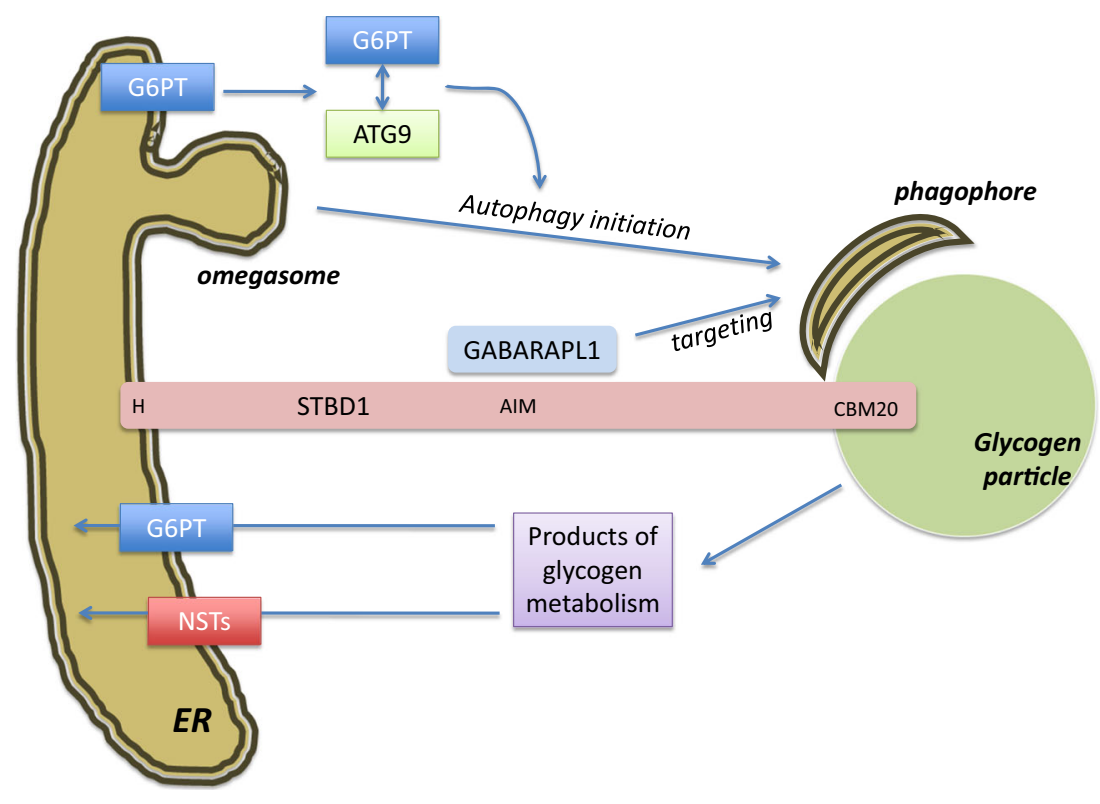


they are mediated by combined enzyme - transporter systems, and the active site of the corresponding enzymes is intraluminal - glucose-6-phosphatase and UDP-glucuronosyl transferases are the best known examples [13-16].

Moreover, ascorbate synthesis (when present) has been shown to be connected to glycogenolysis in the hexuronic acid pathway [17-19] in animals. Gulonolactone oxidase catalysing the last step of ascorbate synthesis in this pathway is expressed also in the ER membrane [20]. Furthermore, several changes in intraluminal redox state are connected to glycogenolysis. Fasting and glucose deprivation decrease the NADPH/NADP redox ratio in the ER lumen via the reduced substrate supply to hexose-6-phosphate dehydrogenase, the main luminal NADPH generating enzyme $[21,22]$ et alet al. It can be supposed that the formation of the carbohydrate moiety of glycoproteins is also connected to glycogen reserves. All the above mentioned metabolic connections were observed in hepatocytes, thus the glycogenoreticular system can be the mechanistic base of a liver-specific metabolic toolkit. Glycogenolysis in glycogen particles is under hormonal control or occurs upon various other stimuli, eg. xenobiotic exposition, intensive ROS formation. Therefore a direct connection among glycogen particles and ER membranes in the liver should be underlying these processes.

Analysis of hepatic glycogen proteome revealed that mitochondrial, peroxisomal, lysosomal and ER proteins are equally present, indicating that glycogen particle specifically interacts with these organelles [6]. Interestingly, glycogen proteome in adipocytes showed a complete absence of ER proteins [23], which suggests that the association of glycogen particles and ER membrane is a liver specific phenomenon [5].

Hepatic glycogen proteome of mice includes proteins that mediate the subcellular localization of glycogen particles, such as starch-binding domain-containing protein 1 (STBD1), which contains both a transmembrane endoplasmic reticulum signal peptide and a carbohydrate-binding domain [6].

Comparing to mouse, the rat glycogen proteome contains even more ER-resident proteins, including cytochrome P450 proteins and UDP-glucuronosyltransferases that are involved in biotransformation [6]. The physical proximity of these proteins with glycogen particles validates the concept of the glycogenoreticular system proposed by us earlier [5] and supports the observation that the glucose unit required for glucuronidation is derived from glycogen and not gluconeogenesis or glucose uptake [11].

\section{Degradation of Glycogen in Autophagosomes}

It is well known that glycogen degradation - glycophagy occurs also in lysosomes (Fig. 1). Recent new observations showed new, interorganelle: glycogen particle - autophagosome connections. These interactions are also serving glucose production for intracellular purposes.

Glycogen can be degraded to glucose by lysosomal or acid alpha-glucosidase in the autophagosomes. Sequestration and degradation of glycogen in the autophagosomes is a selective, hormonally controlled and highly regulated process, representing a mechanism of glucose homeostasis under conditions of demand for the production of this sugar. Targeting of glycogen into the autophagic pathway is governed by two proteins. STBD1 binds glycogen in vitro and in vivo; it is known to anchor glycogen to intracellular membranes [24]. STBD1 also interacts with GABARAPL1, one of the mammalian orthologs of $A \operatorname{tg} 8$, a protein implicated in autophagosome membrane formation and closure in yeast. The LC3-interacting region (LIR) motif of STBD1 binds to GABARAPL1 [25]. This glycogen-specific form of macroautophagy has been designated as glycophagy [24].

Glucose, the final pruduct of lysosomal glycogen hydrolysis, can leave the lumen of the autophagosomes via permeases; spinster (Spin), a putative lysosomal efflux permease has been postulated as a sugar transporter [26]. It was found that the sugar transporter activity of Spin is essential for autophagic lysosome reformation and mTOR reactivation following starvation. Defects in spin led to the accumulation of enlarged autolysosomes. Glucose released into the cytosol can be reutilized in different metabolic pathways following its phosphorylation; alternatively, it can leave the cell contributing to the elevation of blood glucose level.

The importance of glycophagy in the human metabolism has not been revealed in all details. The process evidently has crucial importance in newborns to combat neonatal hypoglycemia by glucagon dependent stimulation of the production of non-phosphorylated glucose from glycogen [27-29]. Animal models revealed that ATG5 and 7 knockout mice with defective autophagy could not survive the first day after birth [30, 31]. Accumulation of glycogen-containing vacuoles (autophagosomes) was observed in the neighbourhood of glycogen granules in the hepatocytes and cardiomyocytes of newborn rats [32, 33].

On the other hand, insulin in the presence of high glucose concentration also resulted in glycogen accumulation and the elevation of glycophagy markers in cultured neonatal rat ventricular myocytes [34]. Indeed, high intracellular glucose concentration was shown to induce autophagy [35]; the effect is possibly mediated by glucose-6-phosphate via decreased phosphorylation of mTOR and Akt.

\section{The Role of the ER in Glycophagy}

Although the localization of the phagophore biogenesis has not been unequivocally clarified, the omegasomes of the ER supposed to be a possible origin of phagophores [36]. With 
respect to glycophagy, two ER proteins seem to participate in the early phase of autophagy. STBD1, which contains both a transmembrane ER signal peptide and a carbohydrate-binding domain [6] has also a LIR domain for binding Atg8-related proteins, such as GABARAPL1 (see above). Thus, the protein has a dual role: it can connect glycogen particle to the ER and can behave as a cargo receptor for glycophagy. Furthermore, the integral ER protein glucose-6-phosphate transporter (G6PT) stimulates the initiation of autophagy by a mechanism which is independent from its transporter activity [37]. G6PT is known to be transcriptionally regulated by a cAMP dependent manner [38], which can promote autophagy in starvation.

\section{Pathological Implications}

Mutations of the already known components of G6Pase system are well characterized and they are known to cause the different subtypes of glycogen storage disease type I (GSD I, von Gierke disease) [39]. Gene defects in lysosomal alpha glucosidase cause GSD type II, also known as Pompe disease, a disorder with autosomal recessive inheritance. The defect of autophagic glycogen degradation leads to the failure of productive autophagy of glycogen in muscle cells. STBD1 could target glycogen molecules for lysosomal recycling either as a random housecleaning process or as part of a more directed transport system. With regard to the latter proposition, it is interesting that STBD1 binds better to plant amylopectin or glycogen from Epm $2 a-/-$ mice. Both amylopectin and Epm 2a-/- glycogen are less branched than normal glycogen, similar to the polyglucosans associated with other glycogen storage diseases, including Andersen disease, Adult polyglucosan disease seen in neurons, glia cells, cardiomyocytes, and Tarui disease [2, 40]. Evidently, aberrantly branched glycogen in cells is to be avoided, and STBD1 could selectively target such abnormal glycogen for transport to lysosomes and for subsequent disposal. It is noted, that STBD1 has higher affinity to structurally abnormal, e.g. less branched glycogen [24]. Another example for polyglucosans is the Lafora disease, where high number of insoluble polyglucosan bodies (Lafora bodies) were shown in neuronal cells [40] leading to chronic neurological diseases.

Galactosamine hepatitis is an experimental glycogen structure abnormality - functional loss example for the ER glycogen particle interactions. In galactosamine- induced liver injury in rats and mice cytoplasmic aggregates containing glycogen, ER membranes and ribosomes were found causing an inhibition of hepatic protein synthesis [41]. Galactosamine incorporated into glycogen causing a structural change, formation of aminoglycogen, which led to formation of these insoluble cytoplasmic bodies.

Autophagy and glycogenolysis compete for glycogen breakdown in the liver. One should suppose a cross- regulation ensuring glucose production in various metabolic conditions, i.e. inhibition of autophagy should stimulate glycogenolysis and vice versa. However, a recent study observed impaired autophagy cultured cells, and animal models of GSD type Ia. Both the stimulation of the anti-autophagic mTOR, and inhibition of the pro-autophagic AMPK pathways were present in various models in vitro and in vivo. Stimulation of autophagy by agents downstream of mTOR and AMPK (ULK1/ATG1 overexpression and rapamycin treatment) decreased hepatic triglyceride and glycogen content in G6PC (-/ -) mice [42]. The results clearly indicate autophagy could partially compensate the metabolic changes in GSD1a, but its inhibion prevents the potential ameliorative effects. Similarly, when autophagy was blocked, the level of glucose-6-phosphatase was reduced in HepG2 cells [43]. Further studies are needed to clarify these virtually conflicting observations, which can be connected to the different functions of autophagy.

\section{Implications in Tumors}

Recycling of macromolecules is a requirement that is particularly important in the tumor microenvironment characterized by low glucose (nutrient?) concentration, hypoxia and acidosis [44]. Glycogen has been reported to be present in different tumor cell lines of various origins in high quantity [45]. However, glycogen metabolism has not been fully elucidated in tumor cells. It can be hypothesized that glycophagy, as a type of autophagy contributes significantly to glycogen recycling in tumors. Tumor cells can have upregulated autophagy [46, 47]; recent findings underline the pro-survival role of autophagy in cellular decision-making processes between life and death both in normal and tumor cells [48, 49]. The demonstration of involvement of glycophagy in the prosurvival effects needs further investigations. These findings suggest that inhibition of the autophagy may threaten tumor survival via metabolic effects, which may open a new approach for cancer therapy [50]. In line with this assumption, combination of caloric restriction with the deficiency in autophagy promotes cell death in tumors [51].

\section{Conclusions}

Glucose is in the focus of metabolism at cellular and multicellular level for obvious reasons: regulation of blood glucose concentration, glucose is the only food molecule for every cell types etc. Mobilization of intracellular energy reserves is a prompt demand upon various stimuli and glycogen particles are essential glucose sources in this sequence of events. Interactions of glycogen particles with the ER or with lysosomes are regulated processes in this organized cellular 
response and all the aspects of selections among these two different routes have not yet explored. It is noted that the liver seems to be unique, as hepatocytes have the full genomic „kit”, which makes possible to perform all kinds of metabolic pathways.

Glycogen degradation has been associated to the energy mobilization in cells for various purposes to different stimuli depending on the cell types. New data on autophagy need a major change in interpretation concerning biological roles of glycogen particles. This progress is also connected on the newly discovered forms of autophagy, as lipophagy and glycophagy, and their relations to the general regulation of cellular growth as mTOR and AMPK. The use of glycogen stores for glucose specific and less glucose specific purposes can be also underlying between the choices of the ways of glycogen degradation. It is noteworthy that from the points of structure-function-metabolism connections macromolecules having altered structures - glycogen, proteins - usually are eliminated by autophagy [52]. On the other hand a comprehensive view on organelle-glycogen particle interactions is also hampered by the relatively limited knowledge on glycogen functions in various other cell types different from hepatocytes and skeletal muscle cells.

\section{References}

1. Adeva-Andany MM, González-Lucán M, Donapetry-García C, Fernández-Fernández C, Ameneiros-Rodríguez E (2016) Glycogen metabolism in humans. Biochem Biophys Acta Clin 5: $85-100$

2. Duran J, Guinovart JJ (2015) Brain glycogen in health and disease. Mol Asp Med 46:70-77

3. Csordas G, Varnai P, Golenar T, Roy S, Purkins G, Schneider TG, Balla T, Hajnoczky G (2010) Imaging interorganelle contacts and local calcium dynamics at the ER-mitochondrial interface. Mol Cell 39:121-132

4. de Brito OM, Scorrano L (2010) An intimate liaison: spatial organization of the endoplasmic reticulum-mitochondria relationship. EMBO J 29:2715-2723

5. Bánhegyi G, Mandl J (2001) The hepatic glycogenoreticular system. POR 7:107-110

6. Stapleton D, Nelson C, Parsawar K, McClain D, Gilbert-Wilson R, Barker E, Rudd B, Brown K, Hendrix W, O'Donnell P, Parker G (2010) Analysis of hepatic glycogen-associated proteins. Proteomics 10:2320-2329

7. Mandl J, Mészáros T, Bánhegyi G, Csala M (2013) Minireview: Endoplasmic reticulum stress: Control in protein, lipid, and signal homeostasis. Mol Endocrinol 27:384-393

8. Csala M, Kereszturi É, Mandl J, Bánhegyi G (2012) The endoplasmic reticulum as the extracellular space inside the cell: role in protein folding and glycosylation. Antioxid Redox Signal 16:11001108

9. Fawcett DW (1955) Observations on the cytology and electron microscopy of hepatic cells. J Natl Cancer Inst 15:1457-1503

10. Cardell RR Jr (1977) Smooth endoplasmic reticulum in rat hepatocytes during glycogen deposition and depletion. Int Rev Cytol 48: 221-279
11. Bánhegyi G, Garzó T, Antoni F, Mandl J (1988) Glycogenolysis and not gluconeogenesis - is the source of UDP-glucuronic acid for glucuronidation. Biochim Biophys Acta 967:429-435

12. Mandl J, Bánhegyi G, Kalapos M, Garzó T (1995) Increased oxidation and decreased conjugation of drugs in the liver caused by starvation. (review) Chem. Biol. Interactions 96:87-101

13. Helmika W, Wever R (1997) A new model for the membrane topology of glucose-6-phosphatase: the enzyme involved in von Gierke disease. FEBS Lett 409:317-319

14. Pan CJ, Lei KJ, Annabi B et al (1998) Transmembrane topology of glucose-6-phosphatase. J Biol Chem 273:6144-6148

15. Burchell G, Coughtrie MW (1989) UDP-glucuronosyltransferases. Pharmacol Ther 43:261-289

16. Clarke DJ, Burchell G (1994) Conjugation-Deconjugation reactions in Drug Metabolism and Toxicity. In: Kauffman FC (ed) Handbook of Experimental Pharmacology, vol 112. Springer Verlag, Budapest, pp 3-43

17. Braun L, Garzo T, Mandl J, Banhegyi G (1994) Ascorbic acid synthesis is stimulated by enhanced glycogenolysis in murine liver. FEBS Lett 352:4-6

18. Braun L, Csala M, Poussu A, Garzo T, Mandl J, Banhegyi G (1996) Glutathione depletion induces glycogenolysis dependent ascorbate synthesis in isolated murine hepatocytes. FEBS Lett 388:173-176

19. Banhegyi G, Braun L, Csala M, Puskas F, Mandl J (1997) Ascorbate metabolism and its regulation in animals. Free Radic Biol Med 23:793-803

20. Puskas F, Braun L, Csala M, Kardon T, Marcolongo P, Benedetti A, Mandl J, Banhegyi G (1998) Gulonolactone oxidase activitydependent intravesicular glutathione oxidation in rat liver microsomes. FEBS Lett 430:293-296

21. Dzyakanchuk AA, Balázs Z, Nashev LG, Amrein KE, Odermatt A (2009) 11beta-Hydroxysteroid dehydrogenase 1 reductase activity is dependent on a high ratio of NADPH/NADP $(+)$ and is stimulated by extracellular glucose. Mol Cell Endocrinol 301:137-141

22. Kereszturi É, Kálmán FS, Kardon T, Csala M, Bánhegyi G (2010) Decreased prereceptorial glucocorticoid activating capacity in starvation due to an oxidative shift of pyridine nucleotides in the endoplasmic reticulum. FEBS Lett 584:4703-4708

23. Stapleton D, Nelson C, Parsawar K, Flores-Opazo M, McClain D, Parker G (2013) The 3T3-L1 adipocyte glycogen proteome. Proteome Sci 22:11

24. Jiang S, Heller B, Tagliabracci VS, Zhai L, Irimia JM, DePaoliRoach AA, Wells CD, Skurat AV, Roach PJ (2010) Starch binding domain-containing protein $1 /$ genethonin 1 is a novel participant in glycogen metabolism. J Biol Chem 285:34960-34971

25. Jiang S, Wells CD, Roach PJ (2011) Starch-binding domain-containing protein 1 (Stbd1) and glycogen metabolism: Identification of the Atg8 family interacting motif (AIM) in Stbd1 required for interaction with GABARAPL1. Biochem Biophys Res Commun 413:420-425

26. Rong Y, McPhee CK, Deng S, Huang L, Chen L, Liu M, Tracy K, Baehrecke EH, Yu L, Lenardo MJ (2011) Spinster is required for autophagic lysosome reformation and mTOR reactivation following starvation. Proc Natl Acad Sci 108:7826-7831

27. Kotoulas OB, Phillips MJ (1971) Fine structural aspects of the mobilization of hepatic glycogen. I. Acceleration of glycogen breakdown. Am J Pathol 63:1-22

28. Schiaffino S, Hanzlíková V (1972) Autophagic degradation of glycogen in skeletal muscles of the newborn rat. J Cell Biol 52:41-51

29. Kotoulas OB, Kalamidas SA, Kondomerkos DJ (2006) Glycogen autophagy in glucose homeostasis. Pathol Res Pract 202:631-638

30. Kuma A, Hatano M, Matsui M, Yamamoto A, Nakaya H, Yoshimori T, Ohsumi Y, Tokuhisa T, Mizushima N (2004) The role of autophagy during the early neonatal starvation period. Nature 432:1032-1036 
31. Komatsu M, Waguri S, Ueno T, Iwata J, Murata S, Tanida I, Ezaki J, Mizushima N, Ohsumi Y, Uchiyama Y, Kominami E, Tanaka K, Chiba T (2005) Impairment of starvation-induced and constitutive autophagy in Atg7-deficient mice. J Cell Biol 169:425-434

32. Kalamidas SA, Kotoulas OB (2000) Glycogen autophagy in newborn rat hepatocytes. Histol Histopathol 15:1011-1018

33. Kondomerkos DJ, Kalamidas SA, Kotoulas OB, Hann AC (2005) Glycogen autophagy in the liver and heart of newborn rats. The effects of glucagon, adrenalin or rapamycin. Histol Histopathol 20:689-696

34. Mellor KM, Varma U, Stapleton DI, Delbridge LM (2014) Cardiomyocyte glycophagy is regulated by insulin and exposure to high extracellular glucose. Am J Physiol Heart Circ Physiol 306: H1240-H1245

35. Ravikumar B, Stewart A, Kita H, Kato K, Duden R, Rubinsztein DC (2003) Raised intracellular glucose concentrations reduce aggregation and cell death caused by mutant huntingtin exon 1 by decreasing mTOR phosphorylation and inducing autophagy. Hum Mol Genet 12:985-994

36. Axe EL, Walker SA, Manifava M, Chandra P, Roderick HL, Habermann A, Griffiths G, Ktistakis NT et al (2008) Autophagosome formation from membrane compartments enriched in phosphatidylinositol 3-phosphate and dynamically connected to the endoplasmic reticulum. J Cell Biol 182:685-701

37. Ahn HH, Oh Y, Lee H, Lee W, Chang JW, Pyo HK, Nah do H, Jung YK (2015) Identification of glucose-6-phosphate transporter as a key regulator functioning at the autophagy initiation step. FEBS Lett 589:2100-2109

38. Chatelain F, Pegorier JP, Minassian C, Bruni N, Tarpin S, Girard J, Mithieux G (1998) Development and regulation of glucose-6phosphatase gene expression in rat liver, intestine, and kidney: in vivo and in vitro studies in cultured fetal hepatocytes. Diabetes 47: 882-889

39. Froissart R, Piraud M, Boudjemline AM, Vianey-Saban C, Petit F, Hubert-Buron A, Eberschweiler PT, Gajdos V, Labrune P (2011) Glucose-6-phosphatase deficiency. Orphanet J Rare Dis 6:27

40. Cavanagh JB (1999) Corpora-amylacea and the family of polyglucosan diseases. Brain Res Brain Res Rev 29:265-295

41. Mandl J, Meszaros K, Antoni F, Spolarics Z, Garzo T (1982) Reversible inhibition of RNA synthesis and irreversible inhibition of protein synthesis by D-galactosamine in isolated mouse hepatocytes. Mol Cell Biochem 46:25-30

42. Farah BL, Landau DJ, Sinha RA, Brooks ED, Wu Y, Fung SY, Tanaka T, Hirayama M, Bay BH, Koeberl DD, Yen PM (2016) Induction of autophagy improves hepatic lipid metabolism in glucose-6-phosphatase deficiency. J Hepatol 64:370-379

43. Jeon JY, Lee H, Park J, Lee M, Park SW, Kim JS, Lee M, Cho B, Kim K, Choi AM, Kim CK, Yun M (2015) The regulation of glucose-6-phosphatase and phosphoenolpyruvate carboxykinase by autophagy in low-glycolytic hepatocellular carcinoma cells. Biochem Biophys Res Commun 463:440-446

44. Kaur J, Debnath J (2015) Autophagy at the crossroads of catabolism and anabolism. Nat Rev Mol Cell Biol 16:461-472

45. Rousset M, Zweibaum A, Fogh J (1981) Presence of glycogen and growth-related variations in 58 cultured human tumor cell lines of various tissue origins. Cancer Res 41:1165-1170

46. Guo JY, Chen HY, Mathew R, Fan J, Strohecker AM, KarsliUzunbas G, Kamphorst JJ, Chen G, Lemons JM, Karantza V et al (2011) Activated Ras requires autophagy to maintain oxidative metabolism and tumorigenesis. Genes Dev 25:460-470

47. Yang S, Wang X, Contino G, Liesa M, Sahin E, Ying H, Bause A, Li Y, Stommel JM, Dell'antonio G, Mautner J, Tonon G, Haigis M, Shirihai OS, Doglioni C, Bardeesy N, Kimmelman AC (2011) Pancreatic cancers require autophagy for tumor growth. Genes Dev 25:717-729

48. Kapuy O, Vinod PK, Mandl J, Bánhegyi G (2013) A cellular stressdirected bistable switch controls the crosstalk between autophagy and apoptosis. Mol BioSyst 9:296-306

49. Das CK, Mandal M, Kögel D (2018) Pro-survival autophagy and cancer cell resistance to therapy. Cancer Metastasis Rev. https://doi. org/10.1007/s10555-018-9727-z

50. Amaravadi R, Kimmelman AC, White E (2016) Recent insights into the function of autophagy in cancer. Genes Dev 30:1913-1930

51. Lashinger LM, O'Flanagan CH, Dunlap SM, Rasmussen AJ, Sweeney S, Guo JY, Lodi A, Tiziani S, White E, Hursting SD (2016) Starving cancer from the outside and inside: Separate and combined effects of calorie restriction and autophagy inhibition on Ras-driven tumors. Cancer Metab 4:18

52. Dikic I (2017) Proteasomal and Autophagic Degradation Systems. Annu Rev Biochem 86:193-224 